\title{
Acute social isolation and regrouping cause short- and long- term molecular changes in the rat medial amygdala
}

\author{
Danit Lavenda-Grosberg ${ }^{1,4}$, Maya Lalzar ${ }^{2,4}$, Noam Leser ${ }^{1}$, Aseel Yaseen ${ }^{1}$, Assaf Malik ${ }^{2}$, Mouna Maroun ${ }^{1}$, Liza Barki-Harrington ${ }^{3,5}$ and $^{2}$ \\ Shlomo Wagner (iD) 1,5 凶
}

(c) The Author(s) 2021

\begin{abstract}
Social isolation poses a severe mental and physiological burden on humans. Most animal models that investigate this effect are based on prolonged isolation, which does not mimic the milder conditions experienced by people in the real world. We show that in adult male rats, acute social isolation causes social memory loss. This memory loss is accompanied by significant changes in the expression of specific mRNAs and proteins in the medial amygdala, a brain structure that is crucial for social memory. These changes particularly involve the neurotrophic signaling and axon guidance pathways that are associated with neuronal network remodeling. Upon regrouping, memory returns, and most molecular changes are reversed within hours. However, the expression of some genes, especially those associated with neurodegenerative diseases remain modified for at least a day longer. These results suggest that acute social isolation and rapid resocialization, as experienced by millions during the COVID-19 pandemic, are sufficient to induce significant changes to neuronal networks, some of which may be pathological.
\end{abstract}

Molecular Psychiatry (2022) 27:886-895; https://doi.org/10.1038/s41380-021-01342-4

\section{INTRODUCTION}

The survival and success of individuals of gregarious mammalian species depend on their ability to form social interactions [1]. These individuals usually habitat in social structures and their wellbeing and survival are threatened by social isolation [2-4]. It is therefore not surprising that in humans, among the most social species on earth $[5,6]$, real or perceived social isolation is associated with compromised health and increased mortality rates [7, 8]. Social isolation also affects mental health [9] and is associates with higher rates of Alzheimer's disease (AD) [10-12]. Actual and perceived loneliness are very common among modern societies [13,14], with as many as $40 \%$ of adults over 65 years of age reporting being lonely at least sometimes [15-17]. Moreover, the recent ongoing COVID-19 pandemic has forced millions around the world into repeated social isolation periods ranging from several weeks to months [18], a situation which may reoccur in future pandemics.

Whereas the psychological effects of social isolation are widely studied, there is much less information about its short- and longterm effects on brain circuitry and activity. While exploration of this important issue in humans presents a major obstacle, mice and rats are optimal models for deciphering the brain mechanisms that are affected by social isolation $[19,20]$. Indeed, the consequences of extended social isolation periods were widely explored in these animal models (Reviewed in [21]). However, there is much less information regarding the consequences of short periods of social isolation (acute social isolation herein) on the brain $[22,23]$. We and others have previously reported that just several days of social isolation significantly impair social recognition memory (SRM) of laboratory rats and mice [20].
Regrouping of the animals after isolation reversed the behavioral effect, suggesting that the brain circuitry has the ability to adapt to changing social conditions [24]. SRM is linked to the medial nucleus of the amygdala (MeA) [25-27], an area that is also involved in human social behavior [28]. Therefore, the purpose of the present study was to identify the molecular and biochemical signatures of the changes that occur in the MeA of adult male rats during acute social isolation and at different time points during regrouping.

\section{RESULTS}

Social isolation and regrouping differentially affect gene expression in the MeA

In order to study the effect of acute social isolation on the MeA, we used a behavioral SRM model, whereby we measured the time spent by an adult male Sprague Dawley rat $(n=10)$ housed in group housing $(\mathrm{G})$ in exploring a same-sex juvenile upon a second encounter (E2). If SRM is formed, the time spent examining the stimulus is significantly reduced compared to the first encounter with the same individual (E1), which took place $2 \mathrm{~h}$ earlier. The results presented in Fig. 1a are in accordance with the ones we previously obtained [24], and show severe impairment of SRM in animals that experienced social isolation for one day (Iso 1d), which lasts throughout seven days of isolation (Iso 7d). One day after returning the animals to group housing (ReGr $1 \mathrm{~d}$ ), SRM is fully restored (2-way repeated ANOVA - encounter $\times$ condition: $F_{(3,27)}=5.368, P=0.005 ;$ post hoc paired $t$-test $-\mathrm{G}: \mathrm{t}_{9}=6.345, p<$ 0.001; ReGr 1d: $\mathrm{t}_{9}=4.088, P=0.007$ ).

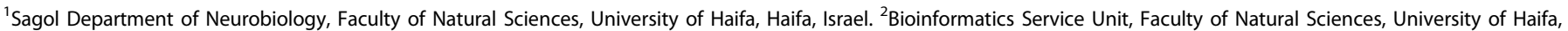

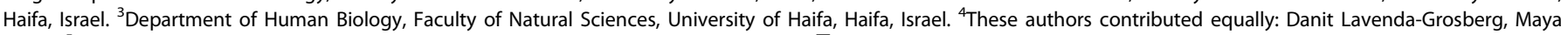
Lalzar. ${ }^{5}$ These authors jointly supervised this work: Liza Barki-Harrington, Shlomo Wagner. ${ }^{凶}$ email: shlomow@research.haifa.ac.il
} 
a

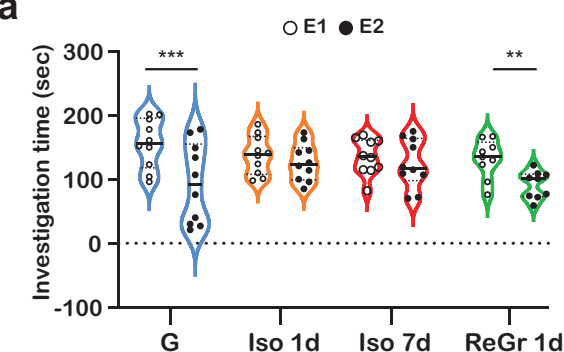

b

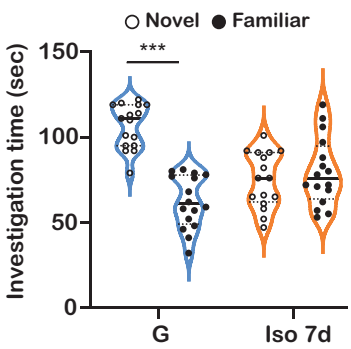

C

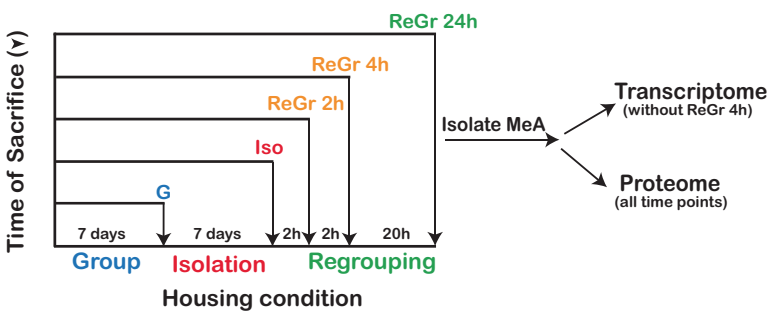

d

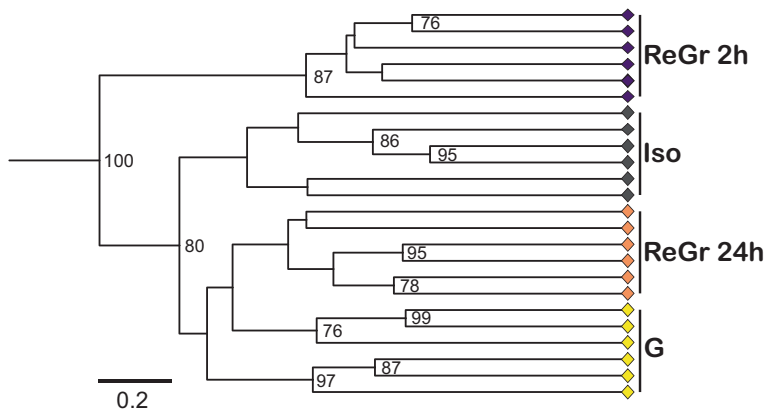

e

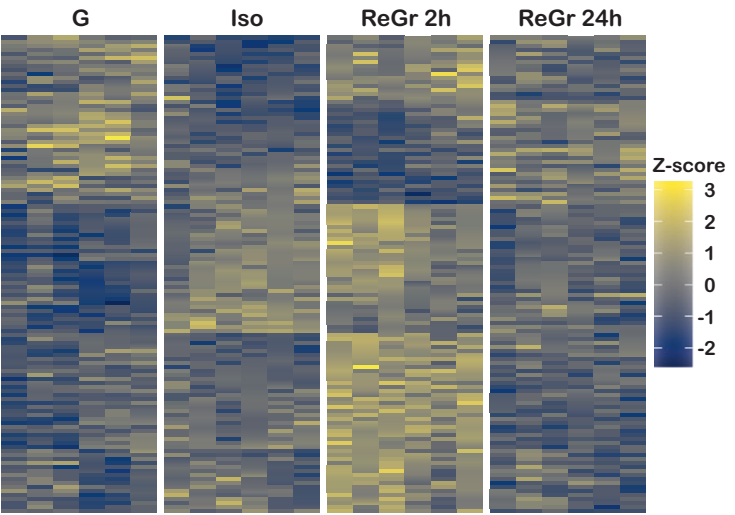

Fig. 1 Social isolation and regrouping differentially affect gene expression in the medial amygdala (MeA). a Social recognition memory (SRM), demonstrated by a reduction in investigation time between two consecutive 5-min encounters (E1, E2) of the subject rat with the same social stimulus, using a 120 min inter-encounter interval. Note the rapid loss of memory one and seven days following isolation (Iso 1d. Iso $7 \mathrm{~d}$ ), compared to group housing $(\mathrm{G})$ and its rapid restoration $1 \mathrm{~d}$ following regrouping (ReGr $1 \mathrm{~d}) .{ }^{* *} p<0.01$, ${ }^{* * *} p<0.001$, paired $t$-test following main effect in 2-way repeated ANOVA. Horizontal lines represent median values. b Social novelty preference of G or Iso $7 \mathrm{~d}$ subjects $(n=16$ per group) towards novel or familiar conspecifics, $24 \mathrm{~h}$ after a 60 min encounter with the familiar conspecific. ${ }^{* * *} p<0.001$, paired $t$-test following main effect in 2-way repeated ANOVA. Horizontal lines represent median values. c Experimental design of omic experiments. Animals were divided into four experimental conditions: grouped (G), isolated for seven days (Iso), isolated for seven days and then regrouped for two hours (ReGr $2 \mathrm{~h}$ ), regrouped for $24 \mathrm{~h}$ following isolation (ReGr $24 \mathrm{~h}$ ). The proteomics experiment had an additional group, of animals regrouped for 4 $\mathrm{h}$ after isolation (ReGr $4 \mathrm{~h}$ ). Following decapitation, MeA samples were extracted and processed for either transcriptomic or proteomic analyses. d Hierarchical clustering according to transcription level of 119 differentially expressed (DE) genes, for the four different experimental conditions, based on Euclidean distance matrix of log-transformed FPKM values. Values at the nodes indicate bootstrap values (as percent of 1000 replications). Bootstrap values below $75 \%$ were omitted. e Heat-maps of a Z-score analysis of the DE genes shown in c, for each animal in the four experimental groups. Z-score was calculated according to the average of all 24 samples (six animals in four groups) per gene.

Previous studies reported that social isolation might increase the motivation of rats for social interactions $[29,30]$. To confirm that our results reflect impaired SRM rather than changes in general motivation for social interactions, we conducted an additional set of social novelty preference experiments, using $G$ and Iso 7d animals. In this paradigm of long-term SRM [27], subject animals were first exposed to a conspecific for $60 \mathrm{~min}$. Twenty four hours later, the subjects were exposed simultaneously to the same conspecific (familiar), as well as a new conspecific (novel), and the time dedicated to their investigation was measured. Statistical analysis revealed a highly significant interaction between individuals (novel vs. familiar) and housing conditions (G vs. Iso 7d) (2-way repeated ANOVA - $F_{(1,30)}=62.57$, $P<0.0001)$. Post hoc analyses revealed that $G$ subjects show significant preference for the novel individual (paired $t$-test, $t_{15}=$ 7.586, $P<0.0001$ ), thus exhibiting intact SRM. In contrast, Iso $7 d$ animals were unable to discriminate between the novel and familiar conspecifics $\left(t_{15}=0.751, P=0.703\right)$, thus confirming that seven days of social isolation impairs SRM (Fig. 1b). No difference was found between the two conditions in the total time the animals spent exploring both stimuli together ( $t$-test, $t_{30}=1.382$,
$P=0.177)$, suggesting no differences in the motivation for social interaction between the two groups.

In order to characterize the transcriptional changes that occur in the rat $\mathrm{MeA}$ in response to acute social isolation and regrouping, we performed an RNA-Seq analysis where animals were assigned one of the following conditions: (1) Grouped (G); (2) Isolated (Iso) that were isolated for seven days; (3) Regrouped for $2 \mathrm{~h}$ (ReGr $2 \mathrm{~h}$ ) that were regrouped for $2 \mathrm{~h}$ following seven days of isolation, and (4) Animals regrouped for $24 \mathrm{~h}$ after isolation (ReGr $24 \mathrm{~h}$ ) (Fig. 1c). The rats used for this analysis did not perform any social behavioral test and were not exposed to a juvenile social stimulus prior to being sacrificed.

The transcriptome analysis identified 16,227 genes (Supplementary Data file 1), 119 of which showed differential expression (DE herein) among the distinct groups (FDR threshold $P<0.05$, FPKM $>0.3, \log _{2} F C> \pm 0.2$; Supplementary Fig. 1). As depicted in Fig. 1d, hierarchical clustering of the DE gene set (Euclidean distances between samples log-transformed FPKM, 1000 bootstrap steps) presented similar gene expression patterns between the $G$ and ReGr $24 \mathrm{~h}$ animals, suggesting that the mRNA levels of most genes return to baseline $24 \mathrm{~h}$ after regrouping. In contrast, 
a

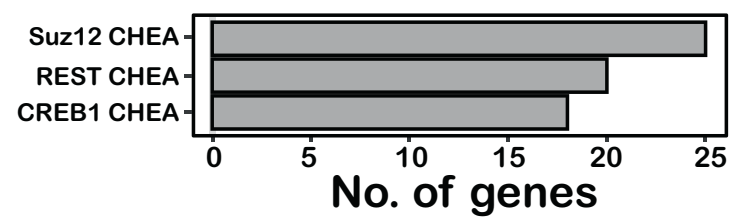

b

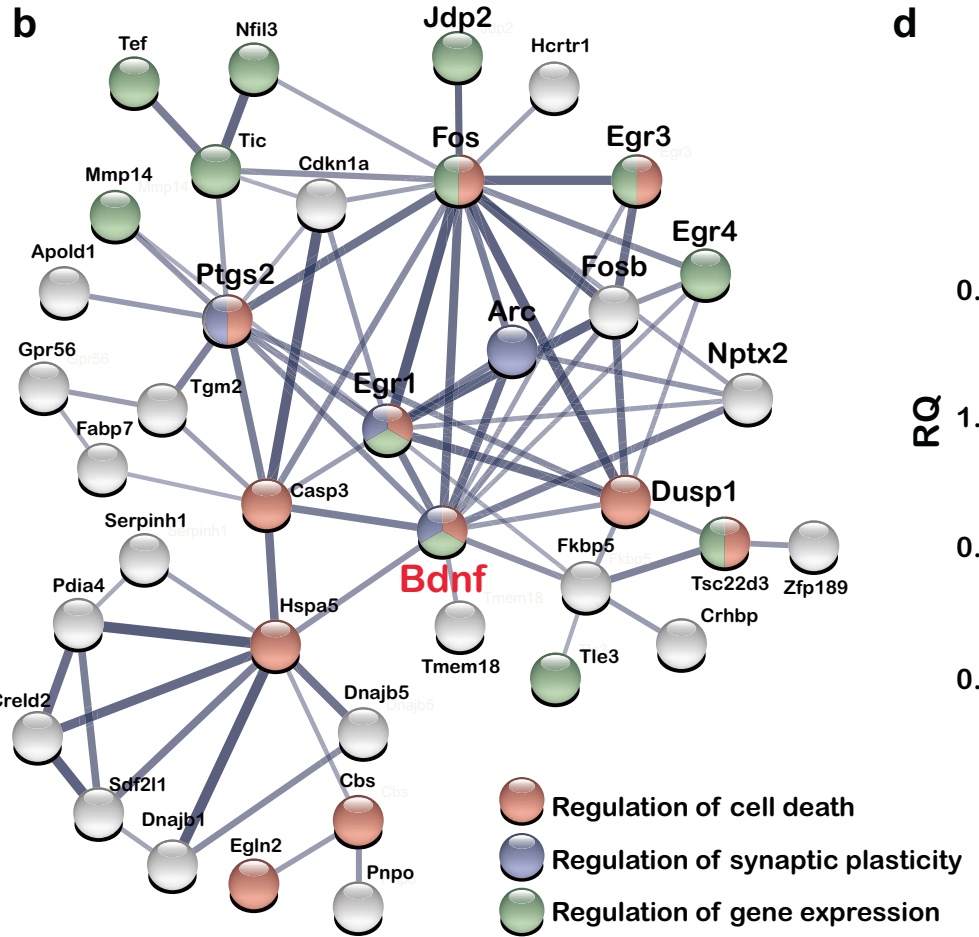

C

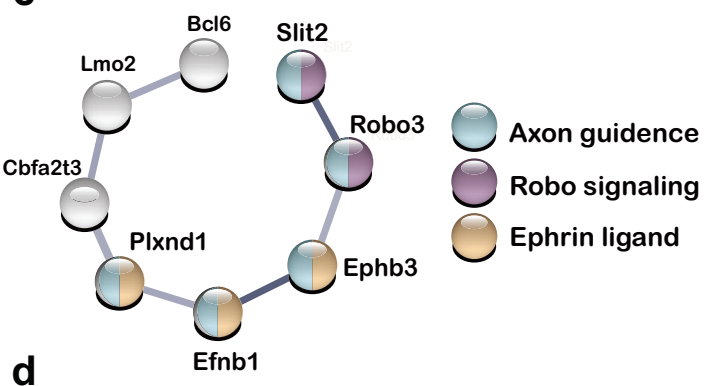

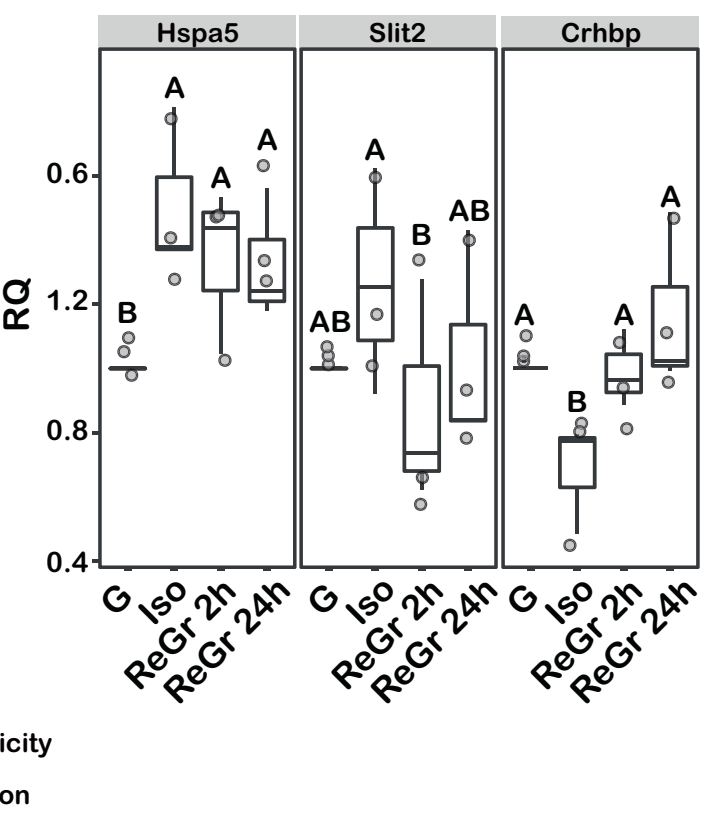

Fig. 2 Differentially expressed (DE) genes are enriched in BDNF signaling and axon guidance pathways. a Functional pathways enriched among DE gene set, queried against the ENCODE CHhA consensus transcription factor (TF) targets from Chip-X database. Presented are TF target sets for which FDR adjusted $P<0.05$. b The largest network revealed by STRING analysis of DE gene set showing a minimum interaction score of medium confidence (0.4). Line thickness indicates the strength of data support. Note that this network is enriched in immediate-early genes (in bold) and genes associated with regulation of cell death, synaptic plasticity and gene expression (color coded according to association). As in c, for the second largest network revealed by STRING analysis. Note that this network is enriched in genes associated with axon guidance, Robo and Ephrin signaling (color coded according to association). d Normalized RQ values of qPCR analysis of three independent sample sets (Exp-1,2,3,) for three genes representing the networks shown in b (Hfspa5, Crhbp) and c (Slit2). Letters represent statistically significant groups.

gene expression in the Iso and ReGr $2 \mathrm{~h}$ groups, showed marked differences compared to the $G$ animals (Fig. 1d). This phenomenon, also apparent in the heat-map of the same gene set (Fig. 1e), suggests that the MeA transcriptome responds rapidly and dynamically to the acute changes in social conditions.

In order to validate the RNA-Seq results, we selected six of the 119 DE genes and used qPCR to measure their mRNA levels in the same samples that were used for the RNA-Seq analysis. We selected three genes that were significantly elevated (FosB, Hspa5, $B d n f$ ) and three that were significantly reduced (Slit2, Mmp14 and Crhbp) between Iso and ReGr $2 \mathrm{~h}$. We also preferred genes that showed a particularly high fold-change (Supplementary Fig. 1a) and were associated with inter-cellular signaling. All genes exhibited a significant correlation (Pearson's correlation, $P<0.05$ ) between the qPCR and RNA-Seq results (Supplementary Fig. 1b, Supplementary Data File 2.

A previous study reported that odor-enriched environment (OEE) rescues SRM in mice isolated for seven days [31]. To examine if a similar effect occurs in rats, we compared the SRM of G rats and Iso 7d rats that were kept in OEE. While there was a borderline significant interaction between encounter and condition (Mixed model ANOVA encounter $\times$ condition: $\left.F_{(1,22)}=4.338, P=0.049\right)$, post hoc analysis revealed that OEE rescued SRM in acutely isolated rats, as both groups showed a significantly reduced investigation time in E2 compared to E1 (post hoc paired $t$-test - G: $t_{10}=5.131, P<0.0001$; Iso $7 \mathrm{~d}: t_{12}=4.836$, $P<0.0001$; Supplementary Fig. 2a). To examine if this OEE-mediated rescue is also reflected in transcriptional changes, we examined one of the above genes, Crhbp, and found no significant difference between the groups ( $t$-test, $t_{10}=1.475, P=0.171$; Supplementary Fig. 2b, Supplementary Data File 2), suggesting that besides SRM, OEE rescues at least some of the transcriptional changes caused by acute isolation.

\section{Social isolation and regrouping affect neuronal network remodeling}

We next used Enrichr [32] to identify the pathways that are enriched among the DE genes (Supplementary Data File 1). This analysis revealed a significant enrichment of genes belonging to the Brain-derived neurotrophic factor (Bdnf) signaling pathway (Benjamini-Hochberg, adj. $P<0.001$ ). Compared to the Iso group, 
a

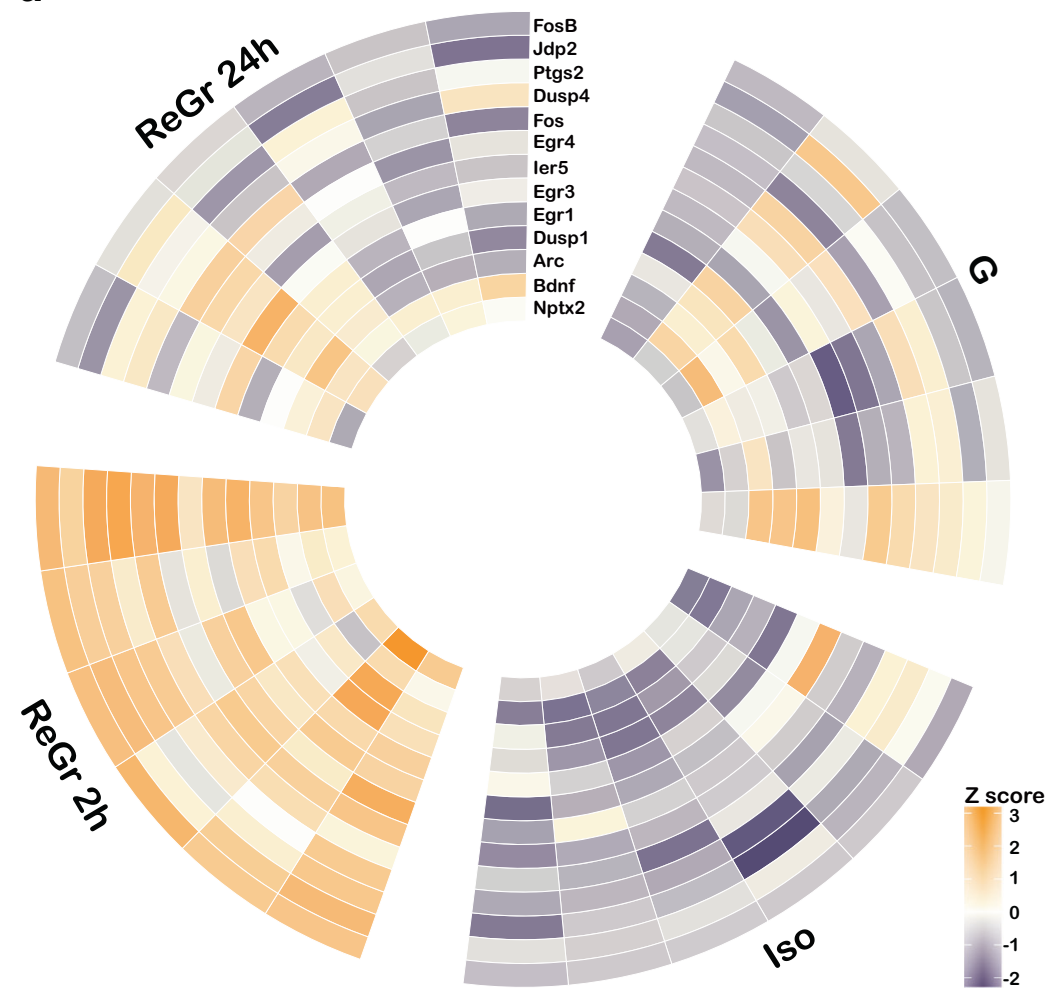

b

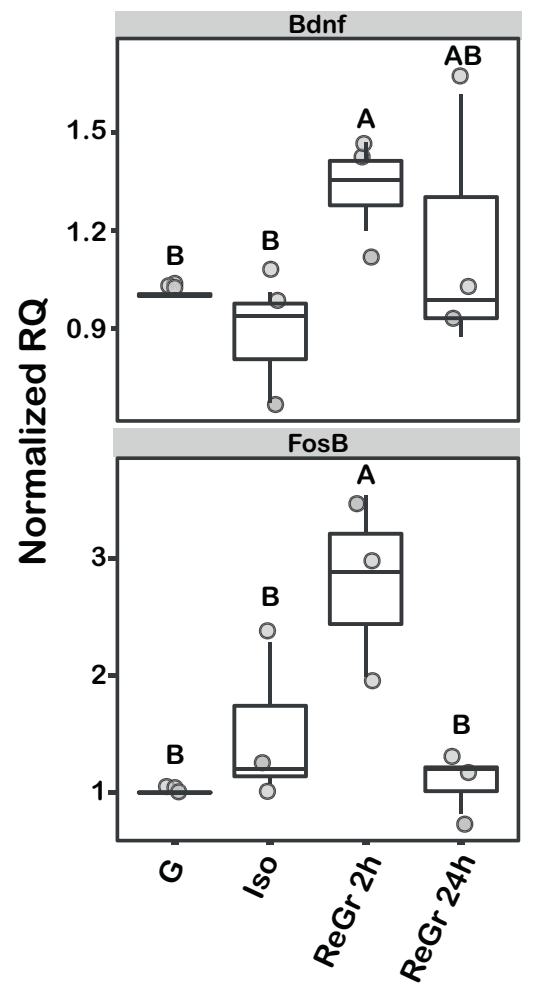

Fig. 3 Regrouping caused a transient elevation in mRNAs of immediate-early genes (IEGs). a A Heat-map of Z-score analysis of 13 IEGs that were differentially expressed between the various conditions. Note that mRNA level of most genes increased two hours after regrouping and returned to baseline $24 \mathrm{~h}$ later. b Normalized RQ values of the three independent sample sets (Exp-1,2,3,) for Bdnf mRNA levels in the MeA (top) and Fosb (bottom). Letters represent statistically significant groups.

the mRNA levels of $B d n f$ itself were significantly upregulated in the ReGr $2 \mathrm{~h}$ and $\operatorname{ReGr} 24 \mathrm{~h}$ animals (log2FC $=0.5, P=0.001$ and $\log 2 \mathrm{FC}=0.49, P=0.004$, respectively). Additional enrichment was found for targets of three transcription factors: Suz12, Rest and Creb1 (Fig. 2a, adj. $P=0.002,0.003$ and 0.002 , respectively), which are associated with brain development [33-37] and memory formation [38, 39].

Application of the STRING database [40] on the DE genes revealed several enriched networks, the largest of which included genes associated with Bdnf signaling, as well as multiple immediate-early genes (Fig. 2b). Additional enriched gene ontologies (GO) were those associated with regulation of cell death (GO: 0010941, FDR adj. $P=0.009$ ), regulation of synaptic plasticity (GO: 0048167, FDR adj. $P=0.013$ ) and regulation of gene expression (GO: 0010468, FDR adj. $P=0.010$ ). The next largest network was that of genes associated with axon guidance including Slit2, Robo3, Ephb3, Efnb1 and others (Fig. 2c).

We next selected the following genes from each network for verification by qPCR: Hspa5 and Crhbp from the Bdnf network, and Slit2 from the axon guidance network. For these experiments, we used the original sample set from the RNA-Seq analysis (Exp-1) and two additional sets obtained from independent experiments (Exp-2, Exp-3). As shown in Fig. 2d, the qPCR experiments confirmed the data obtained by the transcriptome analysis for all genes. Hspa5, also known as BiP or GRP78, is a heat-shock protein who's function is central to ER-associated protein degradation and linked to autophagy/apoptosis processes [41]. The mRNA levels of this gene were consistently higher during isolation (Kruskal-Wallis - H3 $=16.16, P=$ 0.001; Fig. 2d, Supplementary Fig. 3). Similarly, mRNA levels of Slit2, a secreted ligand of Robo receptors that play a central role in axon guidance $[42,43]$, were also markedly elevated in Iso animals and significantly dropped after just two hours of regrouping
(Kruskal-Wallis $-\mathrm{H}_{3}=8.74, P=0.033$; Fig. 2d, Supplementary Fig. 3). Finally, the levels of Crhbp, a brain-wide abundant secreted glycoprotein thought to regulate stress [44], were significantly reduced during isolation and returned to baseline two hours after regrouping (Kruskal-Wallis $-\mathrm{H}_{3}=16.882, P<0.05$; Fig. 2d, Supplementary Fig. 3). Validation of the RNA-Seq data using independent sample sets strengthens our observation that acute isolation and regrouping are accompanied by changes in a specific cohort of signature genes that are associated with neuronal network remodeling such as neurotrophic signaling, synaptic plasticity and axon guidance.

Among the largest network of the DE genes in the STRING analysis, we identified a group of nine immediate-early genes (IEGs, Fig. 2b). Since IEGs are not listed as an ontology, we assembled our own list (Supplementary Data File 1) and used the Chi square test with Yates correction to determine whether they are significantly enriched among the $119 \mathrm{DE}$ genes. This analysis revealed that 11 of the $25 \mathrm{IEGs}$ on our list $\left(44 \%, X^{2}=403.7, P<0.0001\right)$ were significantly upregulated in the ReGr $2 \mathrm{~h}$ compared to the Iso animals (Fig. 3a). To validate these results, we performed qPCR on two genes, Bdnf and FosB, which are common to both the IEGs and the Bdnf signaling pathway. In all three independent experiments (Supplementary Data File 2), the mRNA levels of both genes were significantly higher in the $\operatorname{ReGr} 2 \mathrm{~h}$ compared to both Iso and $G$ animals, with no significant changes between the latter two (Fig. 3b, Supplementary Fig. 3). These results further validate our RNA-Seq analysis and confirm that acute isolation and regrouping lead to induction of specific IEGs and Bdnf signalingassociated genes.

Social isolation and regrouping modify protein expression To determine if acute isolation and regrouping affect protein expression, we prepared another set of MeA samples under the 


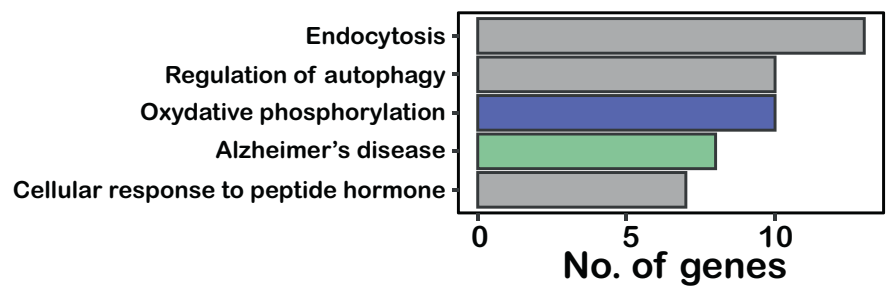

C
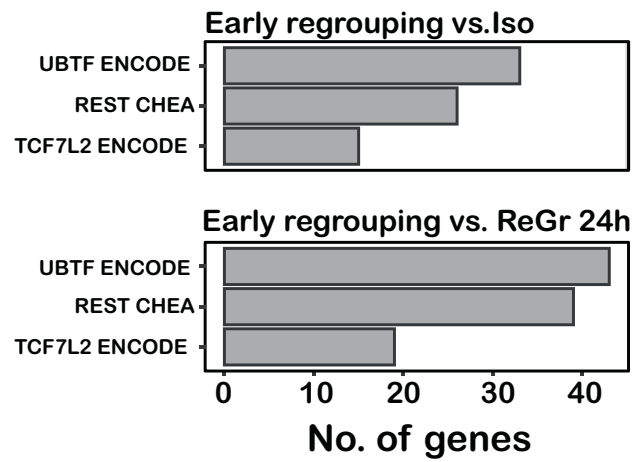

b

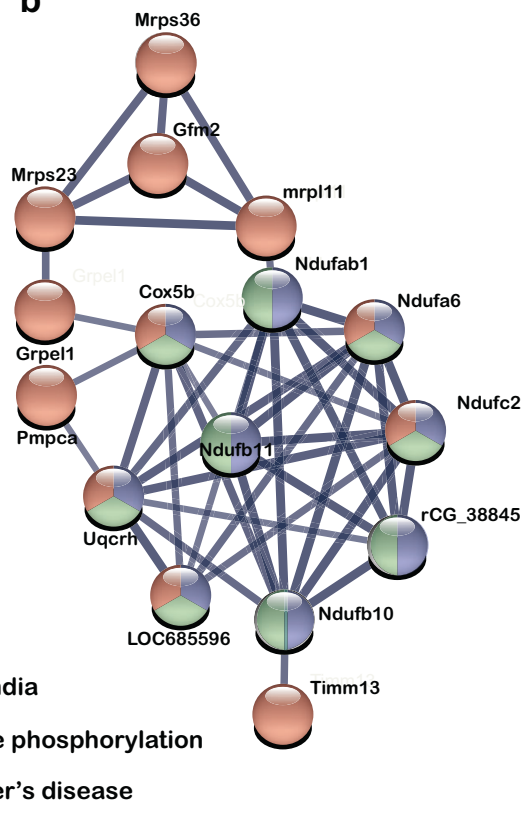

d

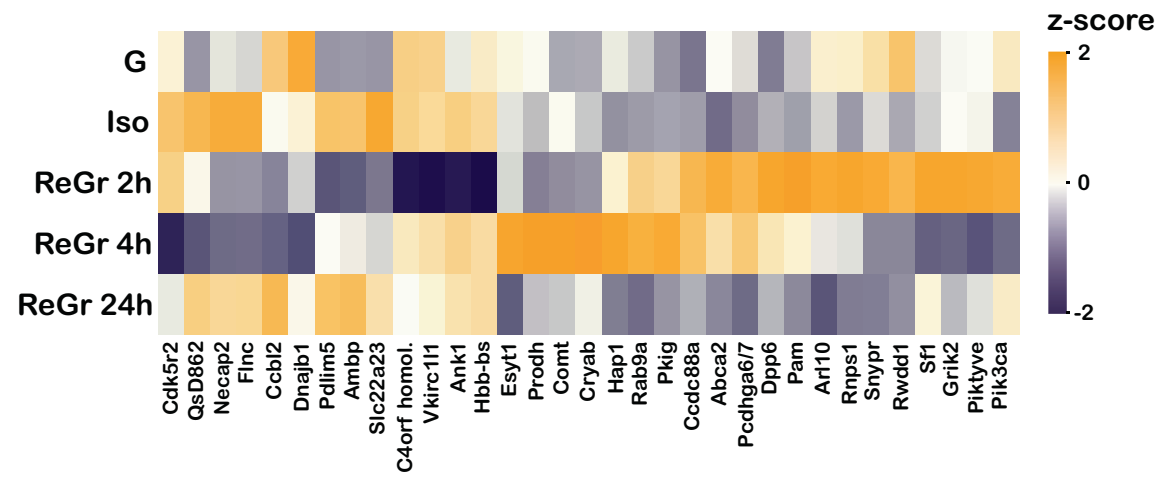

Fig. 4 Proteomic changes in the MeA following isolation and regrouping. a Functional pathways enriched among the 183 proteins that are differentially expressed (adj. $P<0.05$ ) between $G$ and ReGr $24 \mathrm{~h}$ animals, queried against the KEGG and GO databases. $\mathbf{b} \mathrm{A}$ network revealed by STRING analysis of the same set of 183 DE proteins as in a. Line thickness indicates the strength of data support. Note that this network is enriched in proteins associated with the mitochondria, oxidative phosphorylation and Alzheimer's disease (color coded according to association). c Functional pathways enriched among 194 proteins that are differentially expressed between Early regrouping (ReGr $2 \mathrm{~h}$ and ReGr $4 \mathrm{~h}$ ) and Iso animals (upper panel) or ReGr $24 \mathrm{~h}$ animals (lower panel), queried against the ENCODE CHhA consensus transcription factor (TF) targets from Chip-X database. Presented are TF target sets for which FDR adjusted $P<0.05$. $\mathbf{d}$ Heat-map of Z-score analysis of the 33 of the 40 proteins that were differentially expressed in Early regrouping compared to both Iso and ReGr $24 \mathrm{~h}$ animals. These 33 proteins showed opposite trends under the different conditions.

exact experimental conditions described above. Given the significant changes in mRNA expression after $2 \mathrm{~h}$ of regrouping, we reasoned that changes in protein levels may follow, and therefore added another group of animals sacrificed four hours after regrouping (ReGr $4 \mathrm{~h}$ ) (Fig. 1c).

The proteomic analysis identified 4968 expressed proteins, 4767 of which ( 96\%) were also found by the RNA-Seq analysis (Supplementary Data File 3). Compared to $G$ animals, 95 proteins were differentially expressed in the Iso group, 77 of which (80\%) returned to their initial levels in the ReGr $24 \mathrm{~h}$ animals. This suggests that most acute isolation-induced changes in protein levels are transient. However, the levels of 18 proteins remained altered, suggesting that acute isolation may leave a long-term signature. Functional enrichment analysis of these 18 proteins identified the chaperoninmediated protein folding cluster (R-HAS-390466, adj. $P=0.003$ ), including subunits 1 and 4 of Prefoldin, a driver of $A D$ [45]. Among these proteins were also $\mathrm{C} 1 \mathrm{qb}$, Bag5 and Sv2c, which are associated with AD [46-48]. Three additional proteins (Arhgef7, Kalrn, Rgma) belong to the axon guidance pathway (R-HAS-422475, adj. $P=0.044$ ) and three others (Golga2, Snx32, Wdr41) belong to autophagy regulation (GO: 0010506, adj. $P=0.059$ ).

The levels of 183 proteins were markedly changed between the ReGr $24 \mathrm{~h}$ compared to the $\mathrm{G}$ animals, suggesting the long-term effects that are the result of the regrouping process itself. Among these were proteins associated with endocytosis (KEGG: mmu04144. adj. $P=0.0003$ ), oxidative phosphorylation (KEGG: mmu00190, adj. $P=0.0002$ ), neurodegenerative diseases (KEGG: mmu05010, adj. $P$ $=0.0004)$ and regulation of autophagy (GO: 0010506, adj. $P=0.015$ ) (Fig. 4a, Supplementary Data file 3). STRING analysis revealed a dense network of proteins, the core of which were associated with oxidative phosphorylation and mitochondrial electron transport chain, which overlapped with genes associated with AD (Fig. 4b).

To characterize the immediate effects of regrouping, we identified the proteins that changed between the Iso and ReGr $2 \mathrm{~h}$ or ReGr $4 \mathrm{~h}$ 


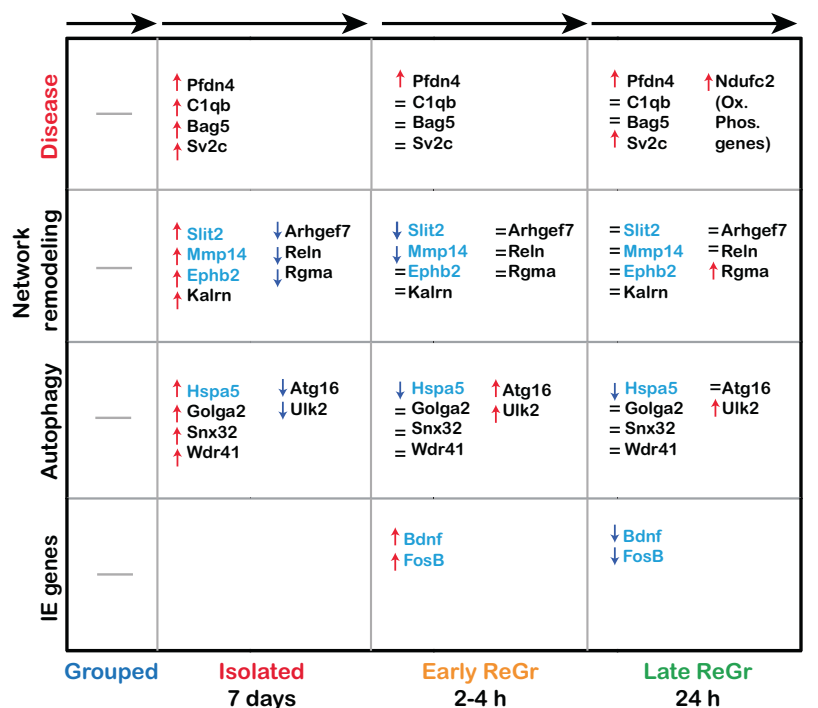

Fig. 5 Functional pathways affected by the various stages of social isolation and regrouping. Overall view of the dynamic molecular changes in the $\mathrm{MeA}$, caused by isolation and regrouping. Shown are four categories: IEGs, Autophagy, Neuronal network remodeling and Disease. Transcriptomic data is marked in light blue and proteomic data - in black. The information in each box refers to change from the previous condition. (=) means no change.

animals (Early Regrouping, herein). The expression of 194 proteins was significantly different between Iso and Early regrouping, 98 were unique to ReGr $2 \mathrm{~h}, 82$ to $\operatorname{ReGr} 4 \mathrm{~h}$ and 14 were common to both, suggesting the existence of highly dynamic changes in protein expression within a very short time frame after regrouping. Functional analysis of the DE proteins in the Early Regrouping samples revealed enrichment of several inter-cellular signaling cascades, including the extracellular signal-regulated kinases (ERK) (BIOCARTA: M6355, adj. $P=0.012$ ), and growth hormone signaling pathways (BIOCARTA: M9043, adj. $P=0.023$ ) (Supplementary Data file 3 ). Analysis of transcription factor targets in the Early Regrouping animals revealed enrichment of targets of several transcription factors including Ubtf, Tcf7l 2 and Rest, all of which are related to neurogenesis and brain development $[33,49,50]$. Targets of these three transcription factors were also enriched among the 225 proteins that were differentially expressed between Early Regrouping and ReGr $24 \mathrm{~h}$ animals (Fig. 4c). Notably, Rest targets were also enriched in the transcriptome analysis (Fig. 2a). These results strongly support the involvement of Ubtf, Tcf7l2 and Rest in the molecular changes that take place in the MeA immediately after regrouping.

To further explore the dynamics in protein expression following regrouping, we identified 40 proteins that changed in Early Regrouping compared to both Iso and ReGr $24 \mathrm{~h}$ animals. Remarkably, 33 of these proteins (83\%) showed opposite trends between the early and late regrouping events (Supplementary Data File 3 ). These proteins represent processes that are strongly affected immediately after regrouping and return to baseline $24 \mathrm{~h}$ later, thus supporting the functionality of the observed proteomic changes (Fig. 4d).

\section{Social isolation and regrouping cause dynamic molecular changes}

A comparison between the proteomic and transcriptomic data yielded only eight differentially expressed genes that were common to both analyses (Crhbp, Synpr, Adgrg1, Dnajb1, Fabp7, Fam107a, Mblac2 and S/c20a2). We therefore reasoned that rather than overlapping, the two analyses are complementary. To examine the overall molecular effect of isolation and regrouping, we combined the results of both analyses into a single list of 812
DE genes/proteins. In terms of signal transduction pathways, this analysis revealed enrichment in two main non-overlapping signaling pathways, namely Bdnf and Pdgf (BioPlanet 2019, adj. $P<0.0001$ for both). Among the different cellular processes that were enriched, the most general ones were endocytosis (KEGG: mmu04144, adj. $P=0.009$ ), autophagy (KEGG: mmu04140, adj. $P=0.016$ ) and axon guidance (R-HAS-422475, adj. $P=0.015$ ). In terms of disease, we found a borderline enrichment in mitochondrial dysfunction associated with neurodegenerative diseases such as AD, Huntington's disease and Amyotrophic Lateral Sclerosis (Elsevier pathway collection, adj. $P=0.076$ ).

To provide an overall view on the dynamics of the molecular changes that occur during isolation and regrouping, we defined four categories of processes that were affected: IEGs (representing rapid signaling events), Autophagy (representing synaptic processes), Network remodeling (representing axon guidance and cell adhesion processes) and Disease. As shown in Fig. 5, IEG levels were increased during Early Regrouping and returned to baseline within $24 \mathrm{~h}$. In contrast, cellular autophagy processes showed a mixed response; while some genes (Golaga2, Snx32 and Wdr42) were upregulated during isolation and remained elevated after regrouping, Ulk2 and Atg16L1 that control autophagosome initiation and nucleation steps [51] were downregulated during isolation and upregulated during regrouping. Interestingly, Hspa5, exhibited opposite trends, with upregulation during isolation and reduction immediately after regrouping. Similarly, network remodeling processes showed elevation in some genes associated with axon guidance during isolation, the levels of which dropped following regrouping (e.g., Slit2, Mmp14). In contrast, other proteins were downregulated during isolation some of which remained low after regrouping (e.g., Arhgef7 and Reln), while others returned to baseline (Rgma). Finally, several proteins that are associated with neurodegenerative diseases were upregulated either during isolation (Pfn4, C1qb, Bag5 and Sv2c) or following regrouping (oxidative phosphorylation-associated genes such as Ndufc2). Together these data suggest that acute social isolation and regrouping induces significant transient changes in the neuronal network of the MeA while leaving a longer lasting molecular signature associated with neurodegenerative diseases.

\section{DISCUSSION}

We have previously demonstrated a severe impairment in SRM of adult rats within just $24 \mathrm{~h}$ of social isolation, which is rapidly reversed following regrouping [24]. SRM was previously shown to be associated with de novo protein synthesis [52]. Here, we studied the molecular changes that occur in the MeA during acute social isolation and regrouping. Our main findings were that each stage has its own molecular signature, and that while most changes are reversible within one day of regrouping, some last longer, particularly those associated with neurodegenerative disorders.

Most studies that explore the effects of social isolation use models of extended isolation ( $>1$ month) that begin immediately after weaning. Such harsh isolation conditions cause various abnormalities [21, 23]. However, they do not mimic the milder types of real or perceived social isolation that are commonly experienced by people [53]. Moreover, these models do not recapitulate much shorter periods of social isolation, such as experienced by millions due to the COVID-19 pandemic [54]. Our model, which better reflects the aforementioned conditions, demonstrates that acute social isolation induces rapid behavioral and molecular effects on the brain. Furthermore, while the SRM is seemingly restored with respect to behavior, acute isolation leaves a long-term molecular signature that may affect the individual later in life. Although our results may be limited to molecular changes and do not reflect anatomical modifications, they suggest 
the occurrence of neuronal network remodeling in the rat MeA in response to acute social deprivation and regrouping.

Two limitations of the study emerge from the time points used for sampling the molecular changes. First, the lack of overlap in DE genes and proteins may stem from the fact that many genes take longer than four hours to express [55]. Second, since we did not investigate a time point that is longer than $24 \mathrm{~h}$, we may have missed some long-term changes that occur later. Nonetheless, our study describes the general dynamics of the molecular changes that occur in the MeA during acute isolation and regrouping, which may leave a persistent mark.

The MeA was previously shown to be involved in molecular processes crucial for SRM formation in both rats and mice [25-27]. As such, it receives social-specific chemosensory information that is necessary for SRM formation [56, 57]. During acute isolation, these signals are markedly reduced, and upon regrouping, become abundant and strong. These fluctuations may explain the vast molecular changes following isolation and regrouping. These changes suggest substantial neuronal network remodeling, which may be analogs to reported anatomical changes in sensory cortical areas following sensory deprivation in adulthood [58, 59]. Nevertheless, we cannot exclude the possibility that these changes may involve additional aspects of social behavior, such as altered social hierarchy due to social isolation and regrouping.

Electrophysiological changes following $24 \mathrm{~h}$ of social isolation were reported in other brain areas. Notably, Matthews et al. [60] showed in mice that acute social isolation raises the neuronal activity in dopaminergic neurons of the dorsal raphe nucleus, which enhance the motivation of the animal to interact with social, but not object stimuli. Our study did not examine social motivation per se, but rather showed that acute isolation directly affects SRM (Fig. 1b). Although we cannot exclude the possibility that social motivation undergoes parallel changes that are separate from those of social memory, our results do not support this possibility for two reasons. First, as depicted in Fig. 1a, there is no change in the investigation time in the first encounter (E1) between grouped, isolated and regrouped animals. Second, the total investigation time of both familiar and novel conspecifics in the social preference experiment was the same in isolated and grouped animals (Fig. 1b). A possible explanation for the differences between the study by Matthews et al. and ours is the use of different models systems (mice vs. rats). We have recently shown that compared to mice, rats present with higher and more immediate motivation for social interactions [61]. Accordingly, a pervious study found that changes in the social motivation of acutely isolated rats are restricted to young age [62].

George et al. [63] examined changes in gene expression 1-2 days after social isolation, in the caudomedial forebrain of Zebra finches [64]. The authors reported molecular changes that are similar to ours, with enrichment in the neurotrophin signaling and axon guidance pathways. Notably, several differentially expressed genes were found to overlap between the two studies, including Egr1, Dusp4, Bdnf and Fkbp5, the latter of which is strongly associated with stress response $[65,66]$. These results may reflect an evolutionarily conserved mechanism of brain molecular responses to social isolation.

Interestingly, our results suggest that many of the molecular changes occur after returning from isolation to group housing. These changes may be divided into two categories. The first category involves mostly Creb1 targets (e.g., FosB, Bdnf) that are strongly elevated two hours following regrouping (Figs. 2 and 3). The second category, which is associated with network remodeling, may also be induced by IEGs associated with long-term plastic changes, (e.g Arc) [67]. This effect may be attributed to two additional transcription factors, Rest and Suz12, the downstream targets of which are enriched within the DE genes (Fig. 2). Rest targets were also enriched among the DE proteins. These transcription factors play significant roles in brain development and in neurodevelopmental disorders $[34,68]$. Suz12 a component of the polycomb repressive complex 2
(PRC2), which has a well-established role in CNS development [69] and neurodegenerative diseases [70], specifically in Huntington's disease [71]. Rest is another epigenetic factor, which besides its role in neurogenesis [72], is implicated in Huntington's disease [73]. Importantly, Rest represses genes that promote cell death and $A D$ and protects neurons from oxidative stress and amyloid $\beta$-protein toxicity [74]. AD and oxidative phosphorylation are ontologies that are enriched among the genes that showed long-term changes following social isolation and regrouping.

Multiple studies (reviewed by Cacioppo et al. [75]), link loneliness to cognitive decline, dementia and $A D$ in elderly people. Our results reveal several possible mechanisms for this link. First, among proteins that changed significantly after $24 \mathrm{~h}$ of regrouping, we found enrichment in genes associated with oxidative phosphorylation and mitochondrial electron transport chain, a pathway strongly linked to $A D$ and other types of neurodegeneration [76]. Interestingly, hippocampal administration of an antioxidant alleviate early cognitive deficits induced by social isolation in a genetic mouse model of $A D$ [77]. Second, the changes we observed in genes associated with Bdnf signaling following isolation and regrouping may be linked to $A D$, as a recent large human study linked between social relationship measures, serum Bdnf levels, and the risk of stroke and dementia [78]. Finally, among the 18 genes that changed during isolation and did not return to baseline following regrouping, were two subunits of the prefoldin complex that is known to inhibit both $A \beta$ fibril formation and a-synuclein aggregation [45].

Overall, our results support the "social homeostasis theory", recently proposed by Mathews and Tye, according to which brain mechanisms adapt in order to maintain a stable level of social interactions [79]. Similar to other homeostatic systems, social hemostasis mechanisms are likely to involve behavioral, physiological and molecular adaptations. In accordance with these theories, our results suggest that social deprivation and a following overload of social stimuli, can induce opposite adaptations not only in neural activity of social behavior associated brain regions, but also in the structure of their neural networks. Thus, short periods of social isolation followed by resocialization, as experienced by million during the current COVID19 pandemic, may have much more significant implications on brain processes than is currently appreciated.

\section{MATERIALS AND METHODS \\ Animals}

Sprague Dawley male rats (adult 7-8 week, 225-249 g or juvenile 3 week, $30-35 \mathrm{~g})$ as well as Wistar-Hola male rats were purchased from Envigo (Rehovot, Israel) and maintained under a 12-h light/dark cycle, at a temperature of $22 \pm 2{ }^{\circ} \mathrm{C}$, with food and water available ad libitum. All experiments were performed according to the National Institutes of Health guide for the care and use of laboratory animals, and approved by the Institutional Animal Care and Use Committee (IACUC) of the University of Haifa.

\section{Behavioral experiments}

Social recognition memory (SRM). The SRM test was conducted as previously described [24]. See Supplementary Methods for further details.

Social novelty preference (SNP). SNP experiments were carried out as previously described [27]. See Supplementary Methods for further details.

Odor-enriched environment (OEE). OEE was established through the addition of commercial fruit odors that are regularly used in the cosmetics and food industry [80] to the home cages of the socially isolated rats. The odors were renewed daily throughout the seven days of isolation, by moving each animal every day to a new cage with a new fresh odor absorbed in its bedding. The mixture of clean bedding $(200 \mathrm{~g})$ and odor ( $30 \mu$ of pure essences in $15 \mathrm{ml}$ ) for each cage was prepared daily, 4 min before exposure.

\section{Brain sampling and processing}

All rats were decapitated and their brains immediately removed, immediately frozen on dry ice and stored at $-80^{\circ} \mathrm{C}$ for until further 
processing. The MeA was bilaterally punched from $400 \mu \mathrm{m}$ slices using a $1 \mathrm{~mm}$ punching needle in a cryostat at $-20^{\circ} \mathrm{C}$. Samples were then placed in Eppendorf tubes and stored at $-80^{\circ} \mathrm{C}$ for until further processing.

RNA extraction for RNA-Seq analysis as was carried out using RNeasy Lipid Tissue Mini Kit (Qiagen), according to the manufacturer's instructions. Concentration of RNA was determined using Nanodrop-1000 (Thermo Scientific). mRNA libraries were prepared using TruSeq RNA Library Prep Kit v2 (Illumina) according to the manufacturer's protocol, and sequenced on HiSeq 2500 (Illumina) $50 \mathrm{bp}$ single read run. Sequences were obtained in fastq format from the TGC Sequencing and Bioinformatics Services, Technion Genome Center, Haifa, Israel.

\section{RNA-Seq data analysis}

Sequences were de-multiplexed by sample based on sample unique barcode. Reads were adapter-trimmed using cutadapt 1.15, then lowquality regions were removed with Trimmomatic 0.3. The filtered dataset was inspected in Fastqc. Illumina reads were mapped to the Rattus_norvegicus.Rnor_6.0.90 assembly downloaded from Ensembl using Star v2.5 (Dobin, et al., 2013). Differential expression analysis was conducted using Bioconductor EdgeR (McCarthy, Chen, \& Smyth, 2012) based on RNA-Seq raw read counts per gene. In EdgeR, within-samples normalization is performed using the TMM algorithm by default. In addition, EdgeR between-samples normalization accounts for differences in library size. Subsequently, the program produces a gene-specific biological variation estimate based on an Empirical Bayes method. Based on this per-gene biological variation estimate, the program allows fitting a specific Generalized Linear Model (GLM) to the gene data, and tests for differential expression. EdgeR GLM is based on a negative binomial distribution function by default. Here, in the GLM, we considered the additive effect of Batch and the Group factors. Expression was considered significantly different for genes with FDR adjusted $P$ value $<0.05$. We further filtered out genes with considerable variation among batches. Gene set enrichment testing was performed with Enrichr [32] quarrying differentially expressed genes against BioPlanet, ENCODE and ChEA consensus transcription factors, GO, KEGG and Reactome databases (accessed May, 2021). Gene association networks were calculated for the differentially expressed gene set using STRING [40] accessed on May 2021. Minimum interaction score was set at 0.4 (medium confidence), excluding interactions from text mining or databases. Line thickness indicates the strength of data support.

\section{Quantitative PCR}

For the two repeated experiments done for verification purposes (Exp-2, Exp-3), RNA extraction was done with Tri-reagent solution (Sigma). Each reaction contained PerfeCTa SYBR green FastMix (Quanta Bioscinces), 10 ng of CDNA (synthesized using the qScript CDNA Synthesis Kit, QuntaBio) and $10 \mu \mathrm{M}$ of each primer (Supplementary Data File 2). SYBR RT PCR reactions were conducted on a StepOne plus Real-Time PCR instrument (Applied Biosystems). See Supplementary Methods for further details.

\section{Protein extraction and proteomic analysis}

Proteomics sequencing was performed by the de Botton Institute of Protein Profiling at the Weizmann Institute for Science (Rehovot, Israel). The proteomic analysis was performed on an independent cohort of animals ( $n=30, n=6$ per group) (see Study design section).

Immediately following MeA removal, $50 \mu \mathrm{l}$ of lysis buffer $(100 \mathrm{mM}$ Tris $\mathrm{pH} 7.6,5 \% \mathrm{SDS}$ ) were added to the microtubes, and samples were homogenized, centrifuged at $16,000 \times G$ at $4{ }^{\circ} \mathrm{C}$, and the lysates were kept at $-80^{\circ} \mathrm{C}$ until further processing. Lysates were subjected to a solution tryptic digestion using the S-Trap method (ProtiFi), followed by a desalting step. The resulting peptides were analyzed using nanoflow liquid chromatography (nanoAcquity) coupled with high-resolution, high mass accuracy mass spectrometry (HF). Each sample was analyzed by the instrument separately, in random order in the discovery mode.

\section{Statistical analysis}

Statistical analyses were carried out with GraphPad Prism 7.04 software for Windows, SPSS v21.0 (IBM) and R (v4.1.0). Specific analyses and tests are described in each section. All $t$-tests were two-sided.

\section{DATA AVAILABILITY}

All data needed to evaluate the conclusions in the paper are present in the paper and/or the Supplementary Materials. The proteomic raw data are available by the
ProteomeXchange Consortium via the PRIDE partner repository with the dataset identifier PXD026871. The transcriptomic raw data are available via NCBI SRA identifier PRJNA734478.

\section{REFERENCES}

1. Snyder-Mackler N, Burger JR, Gaydosh L, Belsky DW, Noppert GA, Campos FA et al. Social determinants of health and survival in humans and other animals. Science. 2020;368:eaax9553.

2. Cacioppo JT, Hawkley LC, Norman GJ, Berntson GG. Social isolation. Ann NY Acad Sci. 2011;1231:17-22.

3. Heinrich LM, Gullone E. The clinical significance of loneliness: a literature review. Clin Psychol Rev. 2006;26:695-718.

4. House JS, Landis KR, Umberson D. Social relationships and health. Science. 1988;241:540-5.

5. Frith $U$, Frith $C$. The social brain: allowing humans to boldly go where no other species has been. Philos Trans R Soc Lond B Biol Sci. 2010;365:165-76.

6. Tomasello M. The ultra-social animal. Eur J Soc Psychol. 2014:44:187-94.

7. Holt-Lunstad J, Smith TB, Layton JB. Social relationships and mortality risk: a meta-analytic review. PLoS Med. 2010;7:e1000316.

8. Steptoe A, Shankar A, Demakakos P, Wardle J. Social isolation, loneliness, and allcause mortality in older men and women. Proc Natl Acad Sci USA 2013;110:5797-801.

9. Cacioppo JT, Hughes ME, Waite LJ, Hawkley LC, Thisted RA. Loneliness as a specific risk factor for depressive symptoms: Cross-sectional and longitudinal analyses. Psychol Aging. 2006;21:140-51.

10. Holwerda TJ, Beekman AT, Deeg DJ, Stek ML, van Tilburg TG, Visser PJ, et al. Increased risk of mortality associated with social isolation in older men: only when feeling lonely? Results from the Amsterdam Study of the Elderly (AMSTEL). Psychol Med. 2012;42:843-53.

11. Perissinotto CM, Stijacic Cenzer I, Covinsky KE. Loneliness in older persons: a predictor of functional decline and death. Arch Intern Med. 2012;172:1078-83.

12. Wilson RS, Krueger KR, Arnold SE, Schneider JA, Kelly JF, Barnes LL, et al. Loneliness and risk of Alzheimer disease. Arch Gen Psychiatry. 2007;64:234-40.

13. McPherson M, Smith-Lovin L, Brashears ME. Social isolation in America: changes in core discussion networks over two decades (vol 71, pg 353, 2006). Am Socio Rev. 2008;73:1022-1022.

14. Victor $C R$, Yang KM. The prevalence of loneliness among adults: a case study of the United Kingdom. J Psychol. 2012;146:85-104.

15. Andersson L. Interdisciplinary study of loneliness - with evaluation of social contacts as a means towards improving competence in old-age. Acta Socio. 1982;25:75-80.

16. Pinquart $M$, Sorensen $S$. Influences on loneliness in older adults: a meta-analysis. Basic Appl Soc Psych. 2001;23:245-66.

17. Weeks DJ. A review of loneliness concepts, with particular reference to old-age. Int J Geriatr Psych. 1994;9:345-55.

18. Manca R, De Marco M, Venneri A. The impact of COVID-19 infection and enforced prolonged social isolation on neuropsychiatric symptoms in older adults with and without dementia: a review. Front Psychiatry. 2020;11:585540.

19. Lukas $M$, de Jong TR. Conspecific interactions in adult laboratory rodents: friends or foes? Curr Top Behav Neurosci. 2017;30:3-24.

20. Leser $\mathrm{N}$, Wagner $\mathrm{S}$. The effects of acute social isolation on long-term social recognition memory. Neurobiol Learn Mem. 2015;124:97-103.

21. Mumtaz F, Khan MI, Zubair M, Dehpour AR. Neurobiology and consequences of social isolation stress in animal model-A comprehensive review. Biomed Pharmacother. 2018;105:1205-22.

22. Arakawa $\mathrm{H}$. Ethological approach to social isolation effects in behavioral studies of laboratory rodents. Behav Brain Res. 2018;341:98-108.

23. Fone KC, Porkess MV. Behavioural and neurochemical effects of post-weaning social isolation in rodents-relevance to developmental neuropsychiatric disorders. Neurosci Biobehav Rev. 2008;32:1087-102.

24. Shahar-Gold H, Gur R, Wagner S. Rapid and reversible impairments of short- and long-term social recognition memory are caused by acute isolation of adult rats via distinct mechanisms. PLoS One. 2013;8:e65085.

25. Choleris E, Little SR, Mong JA, Puram SV, Langer R, Pfaff DW. Microparticle-based delivery of oxytocin receptor antisense DNA in the medial amygdala blocks social recognition in female mice. Proc Natl Acad Sci USA. 2007;104:4670-5.

26. Ferguson JN, Aldag JM, Insel TR, Young LJ. Oxytocin in the medial amygdala is essential for social recognition in the mouse. J Neurosci. 2001;21:8278-85.

27. Gur R, Tendler A, Wagner S. Long-term social recognition memory is mediated by oxytocin-dependent synaptic plasticity in the medial amygdala. Biol Psychiatry. 2014;76:377-86.

28. Atzil S, Touroutoglou A, Rudy T, Salcedo S, Feldman R, Hooker JM, et al. Dopamine in the medial amygdala network mediates human bonding. Proc Natl Acad Sci USA. 2017;114:2361-6. 
29. Niesink RJ, van Ree JM. Short-term isolation increases social interactions of male rats: a parametric analysis. Physiol Behav. 1982;29:819-25.

30. Panksepp J, Beatty WW. Social deprivation and play in rats. Behav Neural Biol. 1980;30:197-206.

31. Gusmao ID, Monteiro BM, Cornelio GO, Fonseca CS, Moraes MF, Pereira GS. Odorenriched environment rescues long-term social memory, but does not improve olfaction in social isolated adult mice. Behav Brain Res. 2012;228:440-6.

32. Kuleshov MV, Jones MR, Rouillard AD, Fernandez NF, Duan $Q$, Wang $Z$, et al. Enrichr: a comprehensive gene set enrichment analysis web server 2016 update. Nucleic Acids Res. 2016;44:W90-97.

33. Ballas N, Mandel G. The many faces of REST oversee epigenetic programming of neuronal genes. Curr Opin Neurobiol. 2005;15:500-6.

34. Desai $D$, Pethe P. Polycomb repressive complex 1: regulators of neurogenesis from embryonic to adult stage. J Cell Physiol. 2020;235:4031-45.

35. He D, Wang J, Lu Y, Deng Y, Zhao C, Xu L, et al. IncRNA functional networks in oligodendrocytes reveal stage-specific myelination control by an IncOL1/Suz12 complex in the CNS. Neuron. 2017:93:362-78.

36. Mampay M, Sheridan GK. REST: an epigenetic regulator of neuronal stress responses in the young and ageing brain. Front Neuroendocrinol. 2019;53:100744.

37. Wang J, Wang J, Yang L, Zhao C, Wu LN, Xu L, et al. CTCF-mediated chromatin looping in EGR2 regulation and SUZ12 recruitment critical for peripheral myelination and repair. Nat Commun. 2020;11:4133.

38. Amidfar M, de Oliveira J, Kucharska E, Budni J, Kim YK. The role of CREB and BDNF in neurobiology and treatment of Alzheimer's disease. Life Sci. 2020;257:118020.

39. Lisman J, Cooper K, Sehgal M, Silva AJ. Memory formation depends on both synapse-specific modifications of synaptic strength and cell-specific increases in excitability. Nat Neurosci. 2018;21:309-14.

40. Szklarczyk D, Gable AL, Lyon D, Junge A, Wyder S, Huerta-Cepas J, et al. STRING v11: protein-protein association networks with increased coverage, supporting functional discovery in genome-wide experimental datasets. Nucleic Acids Res. 2019;47:D607-D613.

41. Wang J, Lee J, Liem D, Ping P. HSPA5 Gene encoding Hsp70 chaperone BiP in the endoplasmic reticulum. Gene. 2017;618:14-23.

42. Blockus H, Chedotal A. Slit-Robo signaling. Development. 2016;143:3037-44.

43. Gonda Y, Namba T, Hanashima C. Beyond Axon guidance: roles of Slit-Robo signaling in neocortical formation. Front Cell Dev Biol. 2020;8:607415.

44. Ketchesin KD, Stinnett GS, Seasholtz AF. Corticotropin-releasing hormone-binding protein and stress: from invertebrates to humans. Stress. 2017;20:449-64.

45. Liang J, Xia L, Oyang L, Lin J, Tan S, Yi P, et al. The functions and mechanisms of prefoldin complex and prefoldin-subunits. Cell Biosci. 2020;10:87.

46. Guo K, Li L, Yin G, Zi X, Liu L. Bag5 protects neuronal cells from amyloid betainduced cell death. J Mol Neurosci. 2015;55:815-20.

47. Johnson SA, Lampert-Etchells M, Pasinetti GM, Rozovsky I, Finch CE. Complement mRNA in the mammalian brain: responses to Alzheimer's disease and experimental brain lesioning. Neurobiol aging. 1992;13:641-8.

48. Stout KA, Dunn AR, Hoffman C, Miller GW. The synaptic vesicle glycoprotein 2: structure, function, and disease relevance. ACS Chem Neurosci. 2019;10:3927-38.

49. Chodelkova O, Masek J, Korinek V, Kozmik Z, Machon O. Tcf7L2 is essential for neurogenesis in the developing mouse neocortex. Neural Dev. https://doi.org/ 10.1186/s13064-018-0107-8. 2018;13:8.

50. Toro C, Hori RT, Malicdan MCV, Tifft CJ, Goldstein A, Gahl WA, et al. A recurrent de novo missense mutation in UBTF causes developmental neuroregression. Hum Mol Genet. 2018;27:1310.

51. Tsapras P, Nezis IP. Caspase involvement in autophagy. Cell Death Differ. 2017;24:1369-79.

52. Richter $\mathrm{K}$, Wolf $\mathrm{G}$, Engelmann M. Social recognition memory requires two stages of protein synthesis in mice. Learn Mem. 2005:12:407-13.

53. Cacioppo JT, Hawkley LC. Perceived social isolation and cognition. Trends Cogn Sci. 2009;13:447-54

54. Smith B, Lim MH. How the COVID-19 pandemic is focusing attention on loneliness and social isolation. Public Health Res Pr. https://doi.org/10.17061/ phrp3022008. 2020;30:3022008.

55. Costa-Mattioli M, Sossin WS, Klann E, Sonenberg N. Translational control of longlasting synaptic plasticity and memory. Neuron. 2009;61:10-26.

56. Pro-Sistiaga P, Mohedano-Moriano A, Ubeda-Banon I, Del Mar Arroyo-Jimenez M, Marcos P, Artacho-Perula E, et al. Convergence of olfactory and vomeronasal projections in the rat basal telencephalon. J Comp Neurol. 2007;504:346-62.

57. Spehr M, Spehr J, Ukhanov K, Kelliher KR, Leinders-Zufall T, Zufall F. Parallel processing of social signals by the mammalian main and accessory olfactory systems. Cell Mol Life Sci. 2006;63:1476-84.

58. Chau LS, Akhtar O, Mohan V, Kondilis A, Galvez R. Rapid adult experiencedependent anatomical plasticity in layer IV of primary somatosensory cortex. Brain Res. 2014;1543:93-100.
59. Miquelajauregui A, Kribakaran S, Mostany R, Badaloni A, Consalez GG, PorteraCailliau C. Layer 4 pyramidal neurons exhibit robust dendritic spine plasticity in vivo after input deprivation. J Neurosci. 2015;35:7287-94.

60. Matthews GA, Nieh EH, Vander Weele CM, Halbert SA, Pradhan RV, Yosafat AS, et al. Dorsal Raphe dopamine neurons represent the experience of social isolation. Cell. 2016;164:617-31.

61. Netser S, Meyer A, Magalnik H, Zylbertal A, de la Zerda SH, Briller M, et al. Distinct dynamics of social motivation drive differential social behavior in laboratory rat and mouse strains. Nat Commun. 2020;11:5908.

62. Varlinskaya El, Spear LP. Social interactions in adolescent and adult SpragueDawley rats: impact of social deprivation and test context familiarity. Behav Brain Res. 2008;188:398-405.

63. George JM, Bell ZW, Condliffe D, Dohrer K, Abaurrea T, Spencer K, et al. Acute social isolation alters neurogenomic state in songbird forebrain. Proc Natl Acad Sci USA. 2020;117:23311-6.

64. Mooney R. The neurobiology of innate and learned vocalizations in rodents and songbirds. Curr Opin Neurobiol. 2020;64:24-31.

65. Hahle A, Merz S, Meyners C, Hausch F. The Many Faces of FKBP51. Biomolecules https://doi.org/10.3390/biom9010035. 2019;9:35.

66. Wang QZ, Shelton RC, Dwivedi Y. Interaction between early-life stress and FKBP5 gene variants in major depressive disorder and post-traumatic stress disorder: a systematic review and meta-analysis. J Affect Disord. 2018;225:422-8.

67. Bramham CR, Messaoudi E. BDNF function in adult synaptic plasticity: the synaptic consolidation hypothesis. Prog Neurobiol. 2005;76:99-125.

68. Hwang JY, Aromolaran KA, Zukin RS. The emerging field of epigenetics in neurodegeneration and neuroprotection. Nat Rev Neurosci. 2017;18:347-61.

69. Liu PP, Xu YJ, Teng ZQ, Liu CM. Polycomb repressive complex 2: emerging roles in the central nervous system. Neuroscientist. 2018;24:208-20.

70. von Schimmelmann M, Feinberg PA, Sullivan JM, Ku SM, Badimon A, Duff MK, et al. Polycomb repressive complex 2 (PRC2) silences genes responsible for neurodegeneration. Nat Neurosci. 2016;19:1321-30.

71. Seong IS, Woda JM, Song JJ, Lloret A, Abeyrathne PD, Woo CJ, et al. Huntingtin facilitates polycomb repressive complex 2. Hum Mol Genet. 2010;19:573-83.

72. Ballas N, Grunseich C, Lu DD, Speh JC, Mandel G. REST and its corepressors mediate plasticity of neuronal gene chromatin throughout neurogenesis. Cell. 2005;121:645-57.

73. Zuccato C, Tartari M, Crotti A, Goffredo D, Valenza M, Conti L, et al. Huntingtin interacts with REST/NRSF to modulate the transcription of NRSE-controlled neuronal genes. Nat Genet. 2003;35:76-83.

74. Lu T, Aron L, Zullo J, Pan Y, Kim H, Chen Y, et al. REST and stress resistance in ageing and Alzheimer's disease. Nature. 2014;507:448-54.

75. Cacioppo S, Capitanio JP, Cacioppo JT. Toward a neurology of loneliness. Psychol Bull. 2014;140:1464-504.

76. Federico A, Cardaioli E, Da Pozzo P, Formichi P, Gallus GN, Radi E. Mitochondria, oxidative stress and neurodegeneration. J Neurol Sci. 2012;322:254-62.

77. Hsiao YH, Kuo JR, Chen SH, Gean PW. Amelioration of social isolation-triggered onset of early Alzheimer's disease-related cognitive deficit by $\mathrm{N}$-acetylcysteine in a transgenic mouse model. Neurobiol Dis. 2012;45:1111-20.

78. Salinas J, Beiser A, Himali JJ, Satizabal CL, Aparicio HJ, Weinstein G, et al. Associations between social relationship measures, serum brain-derived neurotrophic factor, and risk of stroke and dementia. Alzheimers Dement. 2017;3:229-37.

79. Matthews GA, Tye KM. Neural mechanisms of social homeostasis. Ann NY Acad Sci. 2019;1457:5-25.

80. Saar D, Grossman Y, Barkai E. Reduced synaptic facilitation between pyramidal neurons in the piriform cortex after odor learning. J Neurosci. 1999;19:8616-22.

\section{ACKNOWLEDGEMENTS}

We thank Dr. Shai Netser and Dr. Shani Haskal de la Zerda for excellent technical assistance. This study was supported by The Human Frontier Science Program (HFSP grant RGP0019/2015 to SW), the Israel Science Foundation (ISF grants \#1350/12, $1361 / 17$ to SW and \# 2240/19 to LBH), the Ministry of Science, Technology and Space of Israel (Grant \#3-12068 to SW) and the United States-Israel Binational Science Foundation (BSF grant \# 2019186 to SW).

\section{AUTHOR CONTRIBUTIONS}

Study conception and design - LBH, SW; Acquisition of data - DLG, NL, AY; Analysis and interpretation of data - AM, DLG, MM, ML, LBH; Drafting of manuscript - LBH, $\mathrm{ML}$, SW. 
COMPETING INTERESTS

The authors declare no competing interests.

\section{ADDITIONAL INFORMATION}

Supplementary information The online version contains supplementary material available at https://doi.org/10.1038/s41380-021-01342-4.

Correspondence and requests for materials should be addressed to Shlomo Wagner.

Reprints and permission information is available at http://www.nature.com/ reprints

Publisher's note Springer Nature remains neutral with regard to jurisdictional claims in published maps and institutional affiliations.
Open Access This article is licensed under a Creative Commons Attribution 4.0 International License, which permits use, sharing, adaptation, distribution and reproduction in any medium or format, as long as you give appropriate credit to the original author(s) and the source, provide a link to the Creative Commons license, and indicate if changes were made. The images or other third party material in this article are included in the article's Creative Commons license, unless indicated otherwise in a credit line to the material. If material is not included in the article's Creative Commons license and your intended use is not permitted by statutory regulation or exceeds the permitted use, you will need to obtain permission directly from the copyright holder. To view a copy of this license, visit http://creativecommons. org/licenses/by/4.0/.

(c) The Author(s) 2021 